\title{
The tumor suppressor OVCA1 is a short half-life protein degraded by the ubiquitin-proteasome pathway
}

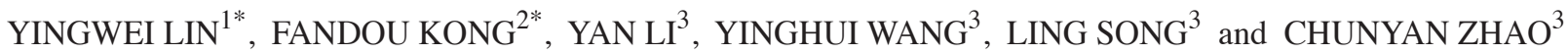 \\ ${ }^{1}$ Department of Clinical Laboratory, The Second Hospital of Dalian Medical University, Dalian, Liaoning 116023; \\ ${ }^{2}$ Department of Gynecology and Obstetrics, The First Affiliated Hospital of Dalian Medical University, Dalian, Liaoning 116011; \\ ${ }^{3}$ College of Laboratory Medicine, Dalian Medical University, Dalian, Liaoning 116044, P.R. China
}

Received April 4, 2018; Accepted November 19, 2018

DOI: $10.3892 / \mathrm{ol} .2018 .9852$

\begin{abstract}
Ovarian cancer gene 1 (OVCA1) is a tumor suppressor associated with ovarian cancer, which is involved in cell proliferation regulation, embryonic development and tumorigenesis. Loss of heterozygosity in the OVCAl gene occurs in $50-86 \%$ of cases of ovarian cancer; however, the physiological and biochemical functions of OVCA1 are not yet clear. In the present study, the stability and degradation of OVCA1 were investigated in A2780, Hela and 293 cells. The results revealed that the OVCA1 protein was unstable by MG132 inhibiting proteasome mediated degradation, co-immunoprecipitation and half-life measurement experiments. The cellular protein levels of endogenous OVCA1 were too low to be detected by western blotting. In addition, carbobenzoxy-L-leucyl-L-leucyl-L-leucinal inhibited the degradation of OVCA1 in cells. The co-immunoprecipitation assay revealed that the OVCA1 protein interacted with ubiquitin to form a poly-ubiquitinated complex in cells. The half-life of OVCA1, measured by inhibiting protein synthesis with cycloheximide, was $<2 \mathrm{~h}$. The present study demonstrated that OVCA1 may be degraded by the ubiquitin-mediated proteasome pathway and may be considered a short half-life protein. In conclusion, the regulation of OVCA1 protein degradation via the ubiquitin-proteasome pathway may represent a novel direction in the development of ovarian cancer therapy.
\end{abstract}

Correspondence to: Professor Chunyan Zhao, College of Laboratory Medicine, Dalian Medical University, 9 West Section, Lvshun South Road, Dalian, Liaoning 116044, P.R. China

E-mail: cyzhao@dlmedu.edu.cn

*Contributed equally

Abbreviations: CHX, cycloheximide; ECL, enhanced chemiluminescence; MG132, carbobenzoxy-L-leucyl-L-leucyl-L-leucinal; UPP, ubiquitin-proteasome pathway; PI, propidium iodide

Key words: co-immunoprecipitation, OVCA1, stability, ubiquitinmediated protein degradation

\section{Introduction}

Ovarian cancer gene $1(O V C A 1)$ is a tumor suppressor gene located on chromosome $17 \mathrm{p} 13.3$ (1). Loss of heterozygosity in the $O V C A 1$ gene occurs in $50-86 \%$ of cases of ovarian cancer (1-5). OVCA1 was originally identified in $1996(1,6)$. Since its sequence is highly similar to that of the yeast diphthamide biosynthesis protein 2, the OVCA1 gene was previously referred to as diphthamide synthesis protein 2-like (7). Previous studies have demonstrated that OVCA1, also termed $\mathrm{DPH1}$ (diphthamide biosynthesis 1), is involved in the biosynthesis of diphthamide by interacting with the eukaryotic translation elongation factor 3 (8-12). OVCA1 has an important role in the regulation of cell proliferation, embryonic development and tumorigenesis (4,13-20). It inhibits the proliferation of epithelial ovarian cancer cells and blocks the cell cycle at $\mathrm{G}_{1}$ phase $(4,19)$ by decreasing cyclin D1 and increasing p16, a tumor suppressor protein (19). Previous studies have demonstrated that OVCAl-mutant mice do not survive during embryonic development or after birth, due to developmental delay and defects in multiple organ systems, and that OVCA1 is involved in p53 deficiency-induced tumorigenesis $(14,18)$. Abnormality in $O V C A 1$ occurs prior to defects in $p 53$ and breast cancer 1 gene, and is therefore considered an early event in ovarian tumorigenesis $(6,14,18,21)$. The close link between $O V C A 1$ and $p 53$ on human chromosome 17 suggests that they may have synchronized effects in cancer development (14). However, the biological function of OVCA1, and its role in tumor occurrence and development have not yet been determined.

To reveal the biological function of OVCA1 and its association with ovarian cancer, commercial antibodies were used to try and detect OVCA1 in cells. However, the results demonstrated that endogenous OVCA1 could not be observed by western blotting with commercial antibodies, despite the large panel of anti-OVCA1 antibodies tested. The mRNA expression levels of $O V C A 1$ were detected by reverse transcription-quantitative polymerase chain reaction (data not shown). Currently, it is well established that the stability of tumor suppressors is positively associated with their functions. In order to explain these phenomena and to further understand the functions of OVCA1, the degradation pathway of OVCA1 and its half-life were investigated in this study. 


\section{Materials and methods}

Cell lines, antibodies, and plasmids. 293, Hela and A2780 cell lines were purchased from the Institute of Shanghai Biochemistry Cell Biology (Shanghai, China). Mouse monoclonal anti-green fluorescence protein (GFP) antibody (cat. no. TA06), mouse monoclonal anti-GAPDH antibody (cat. no. TA08) and horseradish peroxidase labeled goat anti-mouse immunoglobulin $\mathrm{G}$ antibody (catalog no. ZB-2305) were purchased from ZSGB-BIO Company (Beijing, China). Mouse monoclonal anti-ubiquitin antibody (cat. no. sc-8017) was purchased from Santa Cruz Biotechnology, Inc. (Dallas, TX, USA). A GFP-tagged-OVCA1 plasmid was designed by inserting full-length $O V C A 1$ into the XhoI and EcoRI sites of a pEGFP-C1 vector (Clontech Laboratories, Inc., Mountainview, CA, USA).

Cell culture and transfection. Hela and 293 cells were cultured in Dulbecco's modified Eagle's medium (Gibco; Thermo Fisher Scientific, Inc., Waltham, MA, USA) supplemented with $10 \%$ (v/v) newborn calf serum (Gibco; Thermo Fisher Scientific, Inc.) $50 \mathrm{U} / \mathrm{ml}$ penicillin (Gibco; Thermo Fisher Scientific, Inc.), and $50 \mu \mathrm{g} / \mathrm{ml}$ streptomycin (Gibco; Thermo Fisher Scientific, Inc.). A2780 cells were cultured in RPMI-1640 medium (Gibco; Thermo Fisher Scientific, Inc.) supplemented with $10 \%$ (v/v) fetal bovine serum (Gibco; Thermo Fisher Scientific, Inc.), $50 \mathrm{U} / \mathrm{ml}$ penicillin (Invitrogen, Thermo Fisher Scientific, Inc.) and $50 \mu \mathrm{g} / \mathrm{ml}$ streptomycin (Invitrogen, Shanghai, China). Cells were incubated at $37^{\circ} \mathrm{C}$ in a humidified atmosphere containing $5 \% \mathrm{CO}_{2}$. Cells were seeded at a density of $3 \times 10^{5}$ cells/well into 12 -well culture plates and were transfected with Lipofectamine ${ }^{\circledR} 2000$ reagent (Invitrogen; Thermo Fisher Scientific, Inc.), according to the manufacturer's protocol. Briefly, $1 \mu \mathrm{g}$ plasmid DNA was diluted in $50 \mu$ l DMED and $4 \mu 1$ Lipofectamine ${ }^{\circledR} 2000$ reagent was diluted in $50 \mu \mathrm{l}$ DMEM. The diluted plasmid was mixed with the diluted lipofectamine ${ }^{\circledR}$ reagent, and was incubated for $15 \mathrm{~min}$ at temperature. The mixture was added into a $70-90 \%$ confluent cell monolayer. The cells were incubated at $37^{\circ} \mathrm{C}$ in a humidified atmosphere containing $5 \% \mathrm{CO}_{2}$. GFP and GFP-tagged-OVCA1 protein in the cells transfected with empty vector or GFP-tagged-OVCAl were observed directly with a fluorescence microscope.

Cell viability assay. Cell viability was measured by MTT assay. Cells were seeded into 96-well plates (Corning Incorporated, Corning, NY, USA) at $2 \times 10^{4}$ cells/well. After $24 \mathrm{~h}$, cells were treated with various concentrations of carbobenzoxy-L-leuc yl-L-leucyl-L-leucinal (MG132; Merck KGaA, Darmstadt, Germany) for $24 \mathrm{~h}$ at $37^{\circ} \mathrm{C}$ in a humidified atmosphere containing $5 \% \mathrm{CO}_{2}$. Cell viability was assessed using an MTT assay at $490 \mathrm{~nm}$ using a microplate reader.

Flow cytometry. Cells were seeded into 6-well plates (Corning Incorporated, Corning, NY, USA) at $1 \times 10^{6}$ cells/well. After $24 \mathrm{~h}$, cells were treated with various concentrations of MG132 for $24 \mathrm{~h}$ at $37^{\circ} \mathrm{C}$ in a humidified atmosphere containing $5 \%$ $\mathrm{CO}_{2}$. Cells were then fixed with $70 \%$ ethanol at $4^{\circ} \mathrm{C}$ for $12 \mathrm{~h}$, washed with PBS, and resuspended in $500 \mu \mathrm{l}$ PBS containing $45 \mu \mathrm{l}$ RNase A and $405 \mu \mathrm{l}$ propidium iodide (PI) from the Cell
Cycle Detection kit (catalog no. KGA512; Nanjing KeyGen Biotech Co., Ltd., Nanjing, China), and incubated at room temperature for 30-60 min in the dark. Cell proliferation was determined by flow cytometry (BD FACSCalibur; BD Biosciences, Franklin Lakes, NJ, USA).

Western blotting. Cells were lysed in lysis buffer (Beyotime Institute of Biotechnology, Haimen, China) and centrifuged at $12,000 \mathrm{x} \mathrm{g}$ for $5 \mathrm{~min}$. The supernatant was collected as whole cell lysates, and protein concentration was assessed using the Bradford assay (Sigma-Aldrich; Merck KGaA, Darmstadt, Germany). The whole cell lysate $(40 \mu \mathrm{g})$ was separated by $10 \%$ SDS-PAGE and transferred to a polyvinylidene difluoride membrane (Merck KGaA). After $2 \mathrm{~h}$ blocking at room temperature with TBST [20 mmol/1 Tris- $\mathrm{HCl}(\mathrm{pH} 7.4), 137 \mathrm{mmol} / 1$ $\mathrm{NaCl}$ and $0.1 \%$ Tween-20] containing 5\% (w/v) skimmed milk powder, the membranes were incubated with primary antibody (diluted to 1:1,000 with TBST containing 5\% skimmed milk powder) overnight at $4{ }^{\circ} \mathrm{C}$, and were later incubated with horseradish peroxidase-conjugated secondary antibody (diluted to 1:5,000 with TBST containing 5\% skimmed milk powder) for $2 \mathrm{~h}$ at room temperature. An ECL ${ }^{\mathrm{TM}}$ Prime Western Blotting system (catalog no. RPN2232; Merck KGaA) was used to detect protein bands.

Co-immunoprecipitation. Total protein lysate $(200 \mu \mathrm{l})$ was incubated with $2 \mu \mathrm{l}$ anti-GFP monoclonal antibody and $40 \mu \mathrm{l}$ protein $\mathrm{A}+\mathrm{G}$ agarose (Beyotime, Institute of Biotechnology) at $4^{\circ} \mathrm{C}$ for $3 \mathrm{~h}$. After $5 \mathrm{~min}$ centrifugation at 2,500 $\mathrm{x} \mathrm{g}$, the supernatant was discarded and the precipitate was washed five times with PBS and resuspended in $40 \mu 11 \mathrm{X}$ SDS-PAGE loading buffer (Beyotime Institute of Biotechnology). After boiling for $5 \mathrm{~min}$, the supernatant was subjected to immunoblotting.

Half-life measurement. After 24 h of transfection with OVCA1, cells were cultured in medium containing MG132 $(20 \mu \mathrm{mol} / \mathrm{l}$ for Hela and 293 cells, and $5 \mu \mathrm{mol} / 1$ for A2780 cells). After $24 \mathrm{~h}$, cells were cultured in fresh medium containing cycloheximide (CHX; Sigma-Aldrich; Merck KGaA) at a final concentration of $50 \mu \mathrm{g} / \mathrm{ml}$, with or without MG132, for 0, 30, 60, 120, 180, 240 and $300 \mathrm{~min}$, and were subsequently lysed for western blotting. The bands of OVCA1 and GAPDH were scanned, and the intensities of the bands were semi-quantified by ImageJ $1.46 \mathrm{r}$ software (National Institutes of Health, Bethesda, Maryland, USA). The relative concentration of OVCA1 at time 0 was defined as $100 \%$. The percentage of OVCA1 at each indicated time point was normalized by comparing the relative concentration of OVCA1 with that at time 0 . The protein half-life was calculated by linear regression analysis.

Statistical analysis. Data were from at least three independent experiments were expressed as the mean \pm standard deviation. Data were analyzed by one-way analysis of variance followed by Fisher's least significant difference or Tukey's test to compare the means or by bivariate Pearson correlation analysis with SPSS 17.0. (SPSS, Inc., Chicago, IL, USA). P<0.05 was considered to indicate a statistically significant difference. GraphPad Prism version 6 (GraphPad Software, Inc., La Jolla, IL, USA) was used to create the illustrations. 


\section{Results}

Detection of OVCA1 protein in cells. To the best of our knowledge, only six studies have demonstrated the detection of OVCA1 by western blot analysis $(4,8,13,14,18,22)$. Liang et al (18) identified OVCA1 protein in mouse cells by western blotting using a commercial anti-OVCA1 antibody (cat. no. ab40733; Abcam, Cambridge, UK). This antibody and two other anti-OVCA1 antibodies: ab54777 (Abcam), and bs-5714R (BIOSS, Beijing, China) were used in the preliminary experiments. However, no endogenous OVCA1 was observed using these three commercial antibodies by western blotting in any of the cell lines used (Hela, 293 and A2780 cell lines). Subsequently, exogenous GFP-tagged-OVCA1, which was over expressed in Hela cells, was used to assess these three commercial anti-OVCA1 antibodies [cat. nos. ab40733 and ab54777 (Abcam) and BS-5714R (BIOSS)]. After $24 \mathrm{~h}$ transfection, only weak signals in the cells transfected with GFP-tagged-OVCA1 were detected by fluorescence microscopy, and the cell transfection score was lower than in the control cells, which were transfected with a GFP vector only (Fig. 1A). After $48 \mathrm{~h}$ transfection, the fluorescence intensity of the cells transfected with GFP-tagged-OVCA1 was slightly increased compared with at $24 \mathrm{~h}$ (Fig. 1A). Subsequently, the GFP-tagged-OVCA1 protein from these transfected cells was detected by western blotting; however no band was observed after membrane incubation with the aforemention commercial anti-OVCA1 antibodies (data not shown). The membranes were stripped and re-probed with anti-GFP antibody, and a weak band of GFP-tagged-OVCA1 was observed at $75 \mathrm{kD}$ (Fig. 1B). Due to the low level of OVCA1 protein and the high death rate of transfected cells, which was also described by Bruening et al (4), GFP-tagged-OVCA1 was used in this study to help visually estimate the transfection efficiency and the protein expression levels. Small tags, such as hemagglutinin and Myc, cause less disturbance than GFP, but when no or a very weak OVCA1 band is observed, it is difficult to differentiate whether it is a result of high death rate or low transfection efficiency, which may influence the reliability of the results. Therefore in this study, exogenous GFP-OVCA1 was preferentially assessed, and only the results detected with anti-GFP antibody were presented.

OVCA1 degradation is inhibited by MG132. To investigate the pathway of OVCA1 degradation, MG132, a common $26 \mathrm{~S}$ proteasome inhibitor, was used to inhibit the proteasome degradation pathway, which is the main pathway for cellular protein turnover (23). Since MG132 inhibits cell proliferation and induces apoptosis (24-27), the highest concentrations of MG132 that did not influence cell growth were assessed with MTT assay and determined to be $20 \mu \mathrm{mol} / 1$ in Hela and 293 cells, and $5 \mu \mathrm{mol} / 1$ in A2780 cells (Fig. 2Aa). The effects of MG132 on cell proliferation was dose-dependent (Fig. 2Aa). These results were verified by flow cytometry (Fig. 2Ab).

Hela, 293, and A2780 cells were transfected with GFP-tagged-OVCA1. In the absence of MG132 treatment, the fluorescence intensities of GFP-OVCA1 levels were low (Fig. 2B). Following treatment with increasing doses of MG132, the fluorescence intensities of GFP-OVCA1 levels

\section{A}

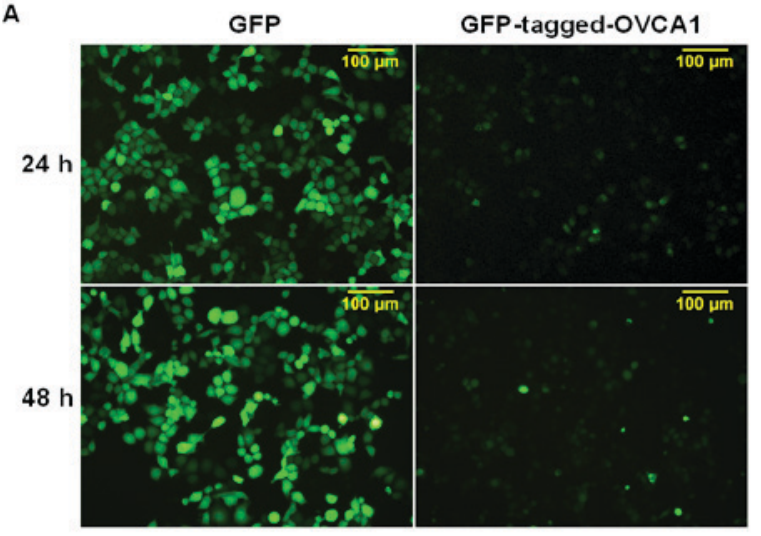

B

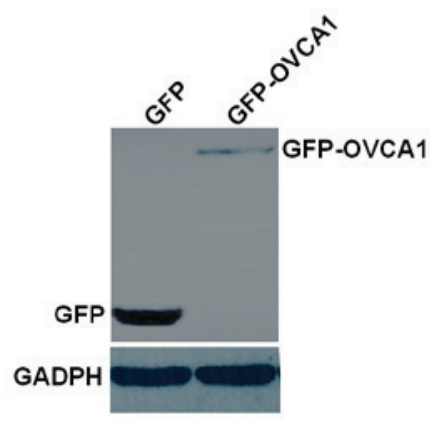

Figure 1. Expression of GFP-tagged-OVCA1 in cells.(A) GFP-tagged-OVCA1 was transfected into Hela cells, and fluorescence intensity was observed by immunofluorescence microscopy 24 or $48 \mathrm{~h}$ post-transfection. (B) GFP-tagged-OVCA1 proteins were detected by western blotting with anti-GFP antibody $48 \mathrm{~h}$ post-transfection. Cells transfected with empty vector were used as controls. Scale bar is $100 \mu \mathrm{m}$. GFP, green fluorescence protein; OVCA1, ovarian cancer gene 1.

were detected and the levels of GFP-tagged-OVCA1 protein in the cells were significantly augmented in a dose-dependent manner (Fig. 2B). The fluorescence intensities and protein levels of GFP in the control cells, which were transfected with empty vector, were not modified following MG132 treatment (Fig. 2C), which was already demonstrated by Gong et al (28). These findings demonstrated that OVCA1 degradation may be inhibited by MG132 in various cell lines.

OVCA1 is degraded by the ubiquitin-proteasome pathway $(U P P)$. To confirm that OVCA1 degradation is mediated by the UPP, the interaction between OVCA1 and ubiquitin was determined by co-immunoprecipitation. Hela, 293 and A2780 cells were transfected with GFP-tagged-OVCA1. To overcome the problem of low GFP-tagged-OVCA1 protein detection, cells treated with MG132 were also used (Fig. 3). GFP-tagged-OVCA1 protein in total cell lysates was pulled down with anti-GFP antibody, and the proteins were analyzed with anti-GFP antibody (Fig. 3A, C and E) to check the pull-down effect. The interaction of GFP-tagged-OVCA1 with ubiquitin was checked with anti-ubiquitin antibody (Fig. 3B, D and F). The protein pull down was also attempted with anti-ubiquitin antibody and by analyzing the interaction with anti-GFP antibody; unfortunately, no band was detected (data not shown). This may be due to a low level of OVCA1 proteins in the cell lysates and therefore, a low percentage of ubiquitinated OVCA1 protein among the pulled-down ubiquitinated proteins. The 
A a

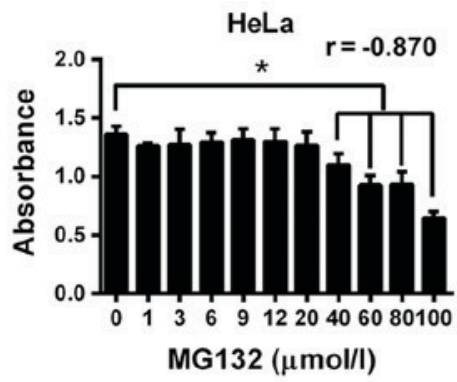

A b

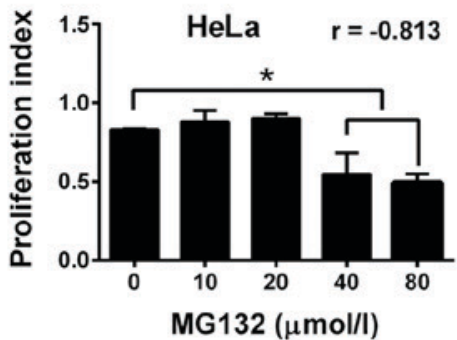

B

MG132

( $\mu \mathrm{mol} / \mathrm{l}$ )

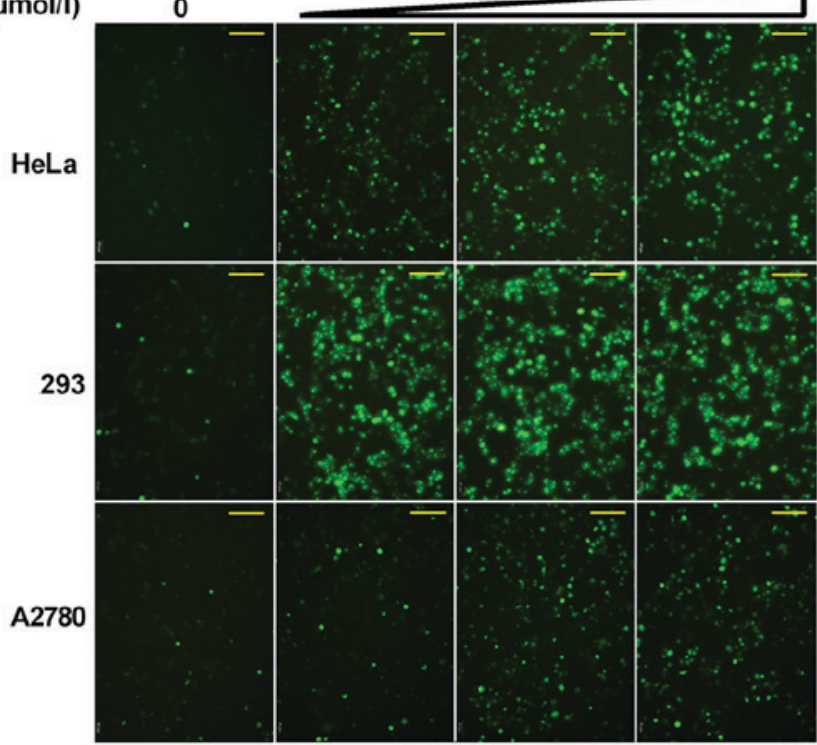

0

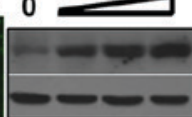

GFP-OVCA 1 GAPDH

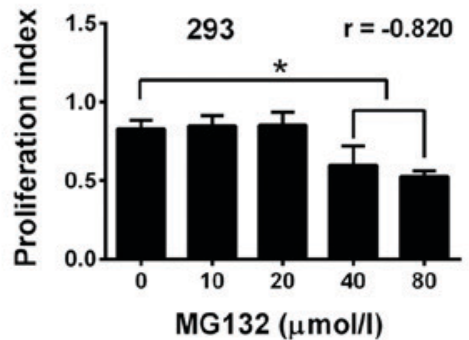

A2780

$r=-0.925$
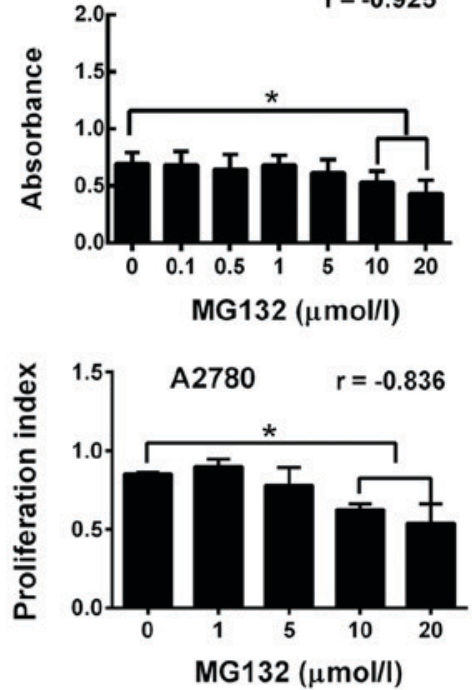
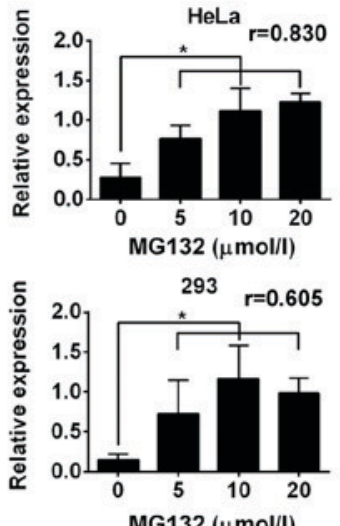

MG132 ( $\mu \mathrm{mol} / \mathrm{l})$
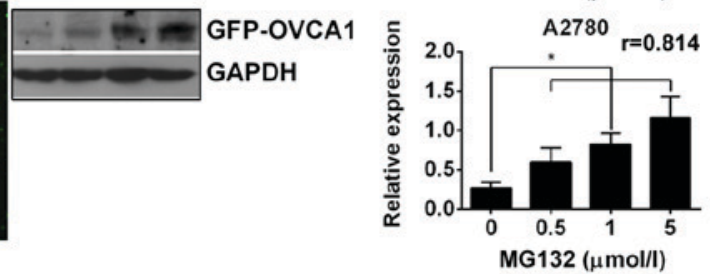

C
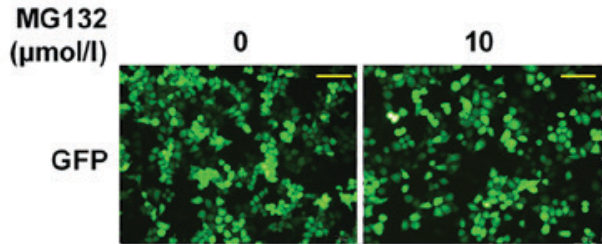

20

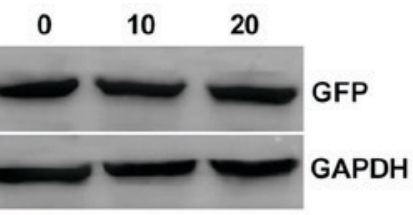

Figure 2. (A) OVCA1 degradation was inhibited by MG132. Cells were seeded and cultured for $24 \mathrm{~h}$ and were treated with various final concentrations of MG132, as indicated, for $24 \mathrm{~h}$. The effects of MG132 on cell proliferation were then detected by (Aa) MTT assay or by (Ab) flow cytometry after propidium iodide staining. Pearson correlation coefficients (r) were indicated in the figure. (B) OVCA1 degradation was blocked by MG132 in a dose-dependent manner. Cells were transfected with GFP-tagged-OVCA1, as indicated. After $24 \mathrm{~h}$, transfected cells were treated with various concentrations of MG132 $(0,5,10$ and $20 \mu \mathrm{mol} / 1$ for Hela and 293 cells; $0,0.5,1$ and $5 \mu \mathrm{mol} / 1$ for A2780 cells) for another $24 \mathrm{~h}$. The fluorescence intensities were observed by fluorescence microscopy and OVCA1 levels were detected by western blotting using an anti-GFP antibody. Relative GFP-tagged-OVCA1 expression was normalized to the internal control GAPDH. (C) GFP protein level was not regulated by MG132. Hela cells were transfected with empty pEGFP-C1 vector. After $24 \mathrm{~h}$, cells were treated with various concentrations of MG132, as indicated, for another $24 \mathrm{~h}$. The fluorescence intensities of GFP were observed by fluorescence microscopy, and GFP protein levels were detected by western blotting. Scale bar, $100 \mu \mathrm{m}$. "P<0.05. GFP, green fluorescence protein; MG132, carbobenzoxy-L -leucyl-L-leucyl-L-leucinal; OVCA1, ovarian cancer gene 1.

results indicated that OVCA1 binds to ubiquitin and forms poly-ubiquitinated OVCA1.

OVCA1 may be a short half-life protein. To reveal the half-life of OVCA1 degradation in cells, CHX, which inhibits protein synthesis in eukaryotic organisms by disturbing the translocation step (29), was used to block the synthesis of proteins in cells. Hela, 293 and A2780 cells were transfected with GFP-tagged-OVCA1. Prior to cell incubation with CHX, cells were treated with MG132, in order to block OVCA1 protein 


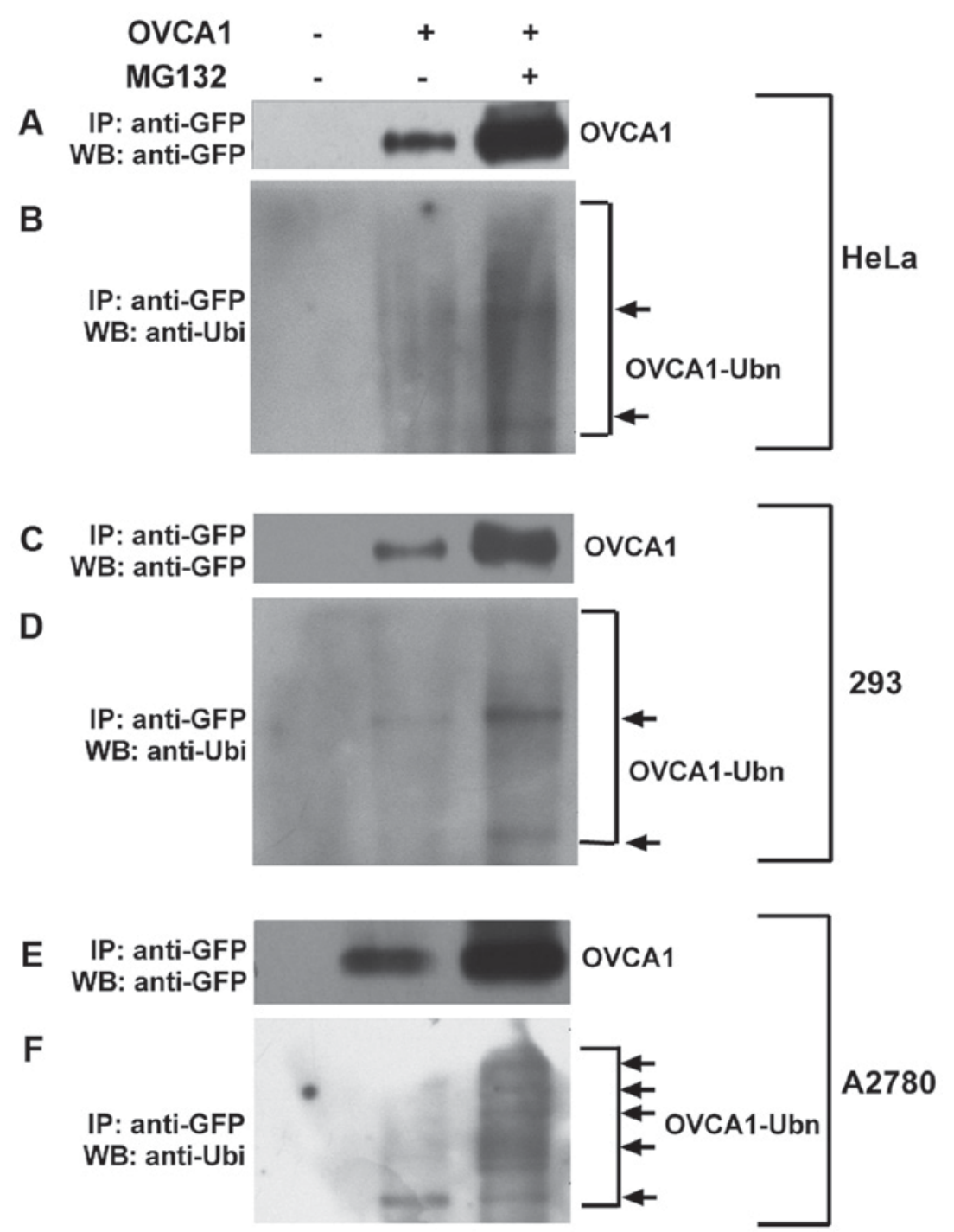

Figure 3. Interaction between OVCA1 and ubiquitin in cells. Cells transfected with GFP-tagged-OVCA1 were treated with or without MG132 as indicated (final concentration of MG132 was $20 \mu \mathrm{mol} / 1$ for Hela and 293 cells, and $5 \mu \mathrm{mol} / 1$ for A2780 cells). Co-IP was then performed. The proteins in total cell lysates were pulled down with anti-GFP antibody. (A, C and E) Pull-down effect; (B, D and F) interaction between GFP-tagged-OVCA1 and ubiquitin. The poly-ubiquitination ladders are indicated with arrowheads. GFP, green fluorescence protein; IP, immunoprecipitation; MG132, carbobenzoxy-L-leucyl-L-leucylL-leucinal; OVCA1, ovarian cancer gene 1; Ubi, ubiquitin; Ubn, polyubiquitin.

degradation and therefore allow the protein detection necessary to carry out the subsequent experiments. After $24 \mathrm{~h}$ of treatment with MG132, cells were treated with $50 \mu \mathrm{g} / \mathrm{ml} \mathrm{CHX}$ alone for $0,30,60,120,180,240$ and $300 \mathrm{~min}$. Cells were treated with CHX and MG132 together for $300 \mathrm{~min}$ as a control. The levels of GFP-tagged-OCVA1 at these time points were detected. Because A2780 cells died during CHX treatment, this experiment was only performed in Hela and 293 cells. The results demonstrated that the protein expression levels of OVCA1 in cells decreased with time and had completely disappeared after 300 min of $\mathrm{CHX}$ treatment. However, when the cells were treated with $\mathrm{CHX}$ and MG132 together for $300 \mathrm{~min}$, the protein expression levels of GFP-tagged-OVCA1 remained high (Fig. 4A), thus suggesting that the loss of GFP-tagged-OVCA1 with time may be caused by ubiquitin-mediated protein degradation. The linear regression analysis demonstrated that the GFP-tagged-OVCA1 half-life was of 105 and 118 min in Hela and 293 cells, respectively (Fig. 4B). These results demonstrated that the OVCA1 protein may have a short half-life.

\section{Discussion}

The deletion or mutation of tumor suppressor genes has an important role in the development of cancer. OVCAl is a tumor suppressor gene, which may be deleted or mutated in ovarian and breast cancer $(3,4,6)$. In addition, the overexpression of $O V C A 1$ inhibits cell proliferation $(4,14,19,20)$. In the present study, the cellular OVCA1 protein levels were demonstrated to be very low. Controlling the stability of cellular proteins is a fundamental way of regulating cell proliferation, survival and development, particularly tumor suppressors. The assessment of a protein half-life is one of the first steps to check whether the function of a protein is regulated by proteolysis under specific physiological conditions. The half-life of OVCA1 measured in this study was 105 and 118 min in Hela and 293 cells, respectively, thus suggesting that OVCA1 may be a short half-life protein. In the present study, the endogenous protein levels of OVCA1 in cells were too low to be detected by western blotting, 

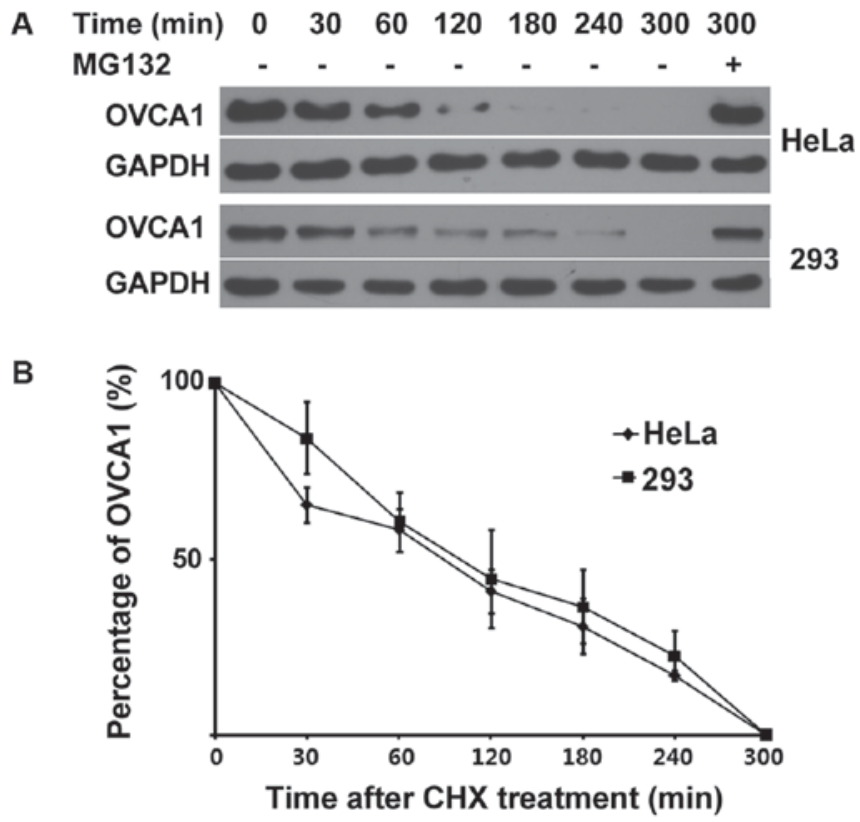

Figure 4.Half-life of OVCA1.Cells were transfected with GFP-tagged-OVCAl and were treated with MG132 at a final concentration of $20 \mu \mathrm{mol} / 1$ for $24 \mathrm{~h}$, prior to treatment with $\mathrm{CHX}$, for the indicated time. (A) Total cell lysates were used for western blotting. (B) Relative concentration of OVCA1 at each indicated time point was calculated. Data were from three independent experiments. CHX, cycloheximide; MG132, carbobenzoxy-L-leucyl-L-leucy 1-L-leucinal; OVCA1, ovarian cancer gene 1.

transfection efficiency was also low and the death rate of OVCAl-transfected cells was high (data not shown). Consequently, the GFP-fused OVCA1 protein was used to observe transfection efficiency and to avoid alterations in OVCA1 protein levels caused by low transfection efficiency or cell death. Although the stability of GFP is not affected by MG132 (28), and it is often used as a tag protein for studies on ubiquitin-mediated protein degradation $(30,31)$, being able to measure either endogenous OVCA1 or a suitable smaller tag fusion protein would be more convincing.

Cellular proteins are degraded through various pathways, including the lysosomal pathway, the UPP and the caspase pathway $(32,33)$. The UPP is a specific protein degradation pathway, which is essentially responsible for the degradation of most intracellular proteins (32). Ubiquitin first attaches to a target protein, involving three critical enzymes, the ubiquitin activating enzyme, the ubiquitin-conjugating enzyme and the ubiquitin ligase, and forms protein complexes. The protein complexes are then recognized by the $26 \mathrm{~S}$ proteasome, a large multi-enzyme complex responsible for protein degradation (34-36). MG132 is an inhibitor commonly used to block the proteolytic activity of the $26 \mathrm{~S}$ proteasome complex (35). In the present study, cellular OVCA1 protein levels were significantly increased following MG132 treatment, thus indicating that OVCA1 may be degraded by the UPP. The subsequent co-immunoprecipitation experiment confirmed that OVCA1 can interact with ubiquitin in cells. These results suggested that the UPP may be one of the pathways allowing the OVCA1 protein degradation.

Modification of ubiquitination serves an important role in regulating protein stability and activity, and is closely associated with the regulation of biological processes and the development of numerous diseases, such as cancer, neurodegenerative diseases and autoimmune diseases (37). The ubiquitin-proteasome system has therefore become an important target for drug screening, and research on the ubiquitination process has become crucial in drug development $(38,39)$. In this study, the OVCA1 was demonstrated to be a short half-life protein that was degraded by the UPP. The molecules involved in the UPP-associated OVCA1 degradation are currently being investigated. Novel molecules targeting the UPP, and hence, regulating the stability or degradation of tumor suppressors have already shown great promise in the treatment of some types of cancer $(38,39)$. Regulating OVCA1 protein degradation may therefore represent a novel target in the treatment of ovarian cancer.

In conclusion, the present study demonstrated that the OVCA1 protein was degraded by the UPP and may be a short half-life protein. These findings provided a potential novel direction for ovarian cancer therapy by regulating OVCA1 protein via UPP. Since endogenous OVCA1 levels were too low to be detected, GFP-tagged OVCA1 was determined in the study. The development of novel techniques for the detection of endogenous OVCA1 is therefore crucial. The signaling pathways of OVCA1 degradation through the UPP will be further investigated in order to provide potential novel targets for the treatment of ovarian cancer.

\section{Acknowledgements}

Not applicable.

\section{Funding}

The present study was supported by the Science and Technology governor of Liaoning Province of China (grant no. 2013023013).

\section{Availability of data and materials}

The datasets used and/or analyzed during the current study are available from the corresponding author on reasonable request.

\section{Authors' contributions}

YWL and FDK participated in the design of the study, the writing and revising of the manuscript, the generation of the figures, cloning of the gene and flow cytometry. YL performed MTT, immunoprecipitation, cloning of the gene and western blotting experiments. YHW partly performed the statistical analysis and flow cytometry, and LS partly performed western blotting experiments. CYZ contributed to the conception and design of the study, was involved in drafting and revising the manuscript, and gave final approval of the manuscript to be published. All authors read and approved the final manuscript.

\section{Ethical approval and consent to participate}

Not applicable.

\section{Patient consent for application}

Not applicable. 


\section{Competing interests}

The authors declare that they have no competing interests.

\section{References}

1. Schultz DC, Vanderveer L, Berman DB, Hamilton TC, Wong AJ and Godwin AK: Identification of two candidate tumor suppressor genes on chromosome 17p13.3. Cancer Res 56: 1997-2002, 1996.

2. Phillips N, Ziegler M, Saha B and Xynos F: Allelic loss on chromosome 17 in human ovarian cancer. Int J Cancer 54: 85-91, 1993.

3. Phillips NJ, Ziegler MR, Radford DM, Fair KL, Steinbrueck T, Xynos FP and Donis-Keller H: Allelic deletion on chromosome 17 p13.3 in early ovarian cancer. Cancer Res 56: 606-611, 1996.

4. Bruening W, Prowse AH, Schultz DC, Holgado-Madruga M, Wong A and Godwin AK: Expression of OVCA1, a candidate tumor suppressor, is reduced in tumors and inhibits growth of ovarian cancer cells. Cancer Res 59: 4973-4983, 1999.

5. Li AJ and Karlan BY: Genetic factors in ovarian carcinoma. Curr Oncol Rep 3: 27-32, 2001.

6. Phillips NJ, Zeigler MR and Deaven LL: A cDNA from the ovarian cancer critical region of deletion on chromosome 17p13.3. Cancer Lett 102: 85-90, 1996.

7. Schultz DC, Balasara BR, Testa JR and Godwin AK: Cloning and localization of a human diphthamide biosynthesis-like protein-2 gene, DPH2L2. Genomics 52: 186-191, 1998.

8. Liu S, Milne GT, Kuremsky JG, Fink GR and Leppla SH: Identification of the proteins required for biosynthesis of diphthamide, the target of bacterial ADP-ribosylating toxins on translation elongation factor 2. Mol Cell Biol 24: 9487-9497, 2004.

9. Mayer K, Schröder A, Schnitger J, Stahl S and Brinkmann U: Influence of DPH1 and DPH5 protein variants on the synthesis of diphthamide, the target of ADPRibosylating toxins. Toxins (Basel) 9: E78, 2017.

10. Stahl S, da Silva Mateus Seidl AR, Ducret A, Kux van Geijtenbeek S, Michel S, Racek T, Birzele F, Haas AK, Rueger R, Gerg M, et al: Loss of diphthamide pre-activates NF- $\mathrm{KB}$ and death receptor pathways and renders MCF7 cells hypersensitive to tumor necrosis factor. Proc Natl Acad Sci USA 112: 10732-10737, 2015

11. Webb TR, Cross SH, McKie L, Edgar R, Vizor L, Harrison J, Peters J and Jackson IJ: Diphthamide modification of eEF2 requires a J-domain protein and is essential for normal development. J Cell Sci 121: 3140-3145, 2008

12. Nobukuni Y, Kohno K and Miyagawa K: Gene trap mutagenesis-based forward genetic approach reveals that the tumor suppressor OVCA1 is a component of the biosynthetic pathway of diphthamide on elongation factor 2. J Biol Chem 280 : 10572-10577, 2005.

13. Chen CM and Behringer RR: Cloning, structure, and expression of the mouse Ovcal gene. Biochem Biophys Res Commun 286 1019-1026, 2001

14. Chen CM and Behringer RR: Ovca1 regulates cell proliferation, embryonic development, and tumorigenesis. Genes Dev 18 : 320-332, 2004

15. Jensen MR and Helin K: OVCA1: Emerging as a bona fide tumor suppressor. Genes Dev 18: 245-248, 2004.

16. L'Allemain G: Ovcal gene, deleted in ovarian cancer is a special tumor suppressor. Bull Cancer 91: 301-302, 2004 (In French).

17. Chen CM and Behringer RR: OVCA1: Tumor suppressor gene. Curr Opin Genet Dev 15: 49-54, 2005.

18. Liang M, Ayanga B, Du S, Godwin AK, Hartsock JK and Evans SC: Ovca1, a candidate gene of the genetic modifier of Tp53, Mop2, affects mouse embryonic lethality. Genes Chromosomes Cancer 47: 315-325, 2008.

19. Kong F, Tong R, Jia L, Wei W, Miao X, Zhao X, Sun W, Yang G and Zhao C: OVCA1 inhibits the proliferation of epithelial ovarian cancer cells by decreasing cyclin D1 and increasing p16. Mol Cell Biochem 354: 199-205, 2011.
20. Yu YR, You LR, Yan YT and Chen CM: Role of OVCA1/DPH1 in craniofacial abnormalities of Miller-Dieker syndrome. Hum Mol Genet 23: 5579-5596, 2014.

21. Wiper DW, Zanotti KM, Kennedy AW, Belinson JL and Casey G: Analysis of allelic imbalance on chromosome $17 \mathrm{p} 13$ in stage I and stage II epithelial ovarian cancers. Gynecol Oncol 71: 77-82, 1998.

22. Prowse AH, Vanderveer L, Milling SW, Pan ZZ, Dunbrack RL, $\mathrm{Xu}$ XX and Godwin AK: OVCA2 is downregulated and degraded during retinoid-induced apoptosis. Int J Cancer 99: 185-192, 2002.

23. Rock KL, Gramm C, Rothstein L, Clark K, Stein R, Dick L, Hwang D and Goldberg AL: Inhibitors of the proteasome block the degradation of most cell-proteins and the generation of peptides presented on MHC class-I molecules. Cell 78: 761-771, 1994.

24. Jullig M, Zhang WV, Ferreira A and Stott NS: MG132 induced apoptosis is associated with p53-independent induction of pro-apoptotic Noxa and transcriptional activity of beta-catenin. Apoptosis 11: 627-641, 2006.

25. Han YH, Moon HJ, You BR and Park WH: The effect of MG132, a proteasome inhibitor on HeLa cells in relation to cell growth, reactive oxygen species and GSH. Oncol Rep 22: 215-221, 2009.

26. Han YH and Park WH: MG132 as a proteasome inhibitor induces cell growth inhibition and cell death in A549 lung cancer cells via influencing reactive oxygen species and GSH level. Hum Exp Toxicol 29: 607-614, 2010.

27. Guo N and Peng Z: MG132, a proteasome inhibitor, induces apoptosis in tumor cells. Asia Pac J Clin Oncol 9: 6-11, 2013.

28. Gong Y, Wang D, Dar JA, Singh P, Graham L, Liu W, Ai J, Xin Z, Guo Y and Wang Z: Nuclear export signal of androgen receptor (NESAR) regulation of androgen receptor level in human prostate cell lines via ubiquitination and proteasome-dependent degradation. Endocrinology 153: 5716-5725, 2012.

29. Watanabe-Asano T, Kuma A and Mizushima N: Cycloheximide inhibits starvation-induced autophagy through mTORC1 activation. Biochem Biophys Res Commun 445: 334-339, 2014.

30. Fu C, Chen D, Chen R, Hu Q and Wang G: The schizophrenia-related protein dysbindin-1A is degraded and facilitates NF-Kappa B activity in the nucleus. PLoS One 10: e0132639, 2015.

31. Nakagawa $K$ and Yokosawa $H$ : Degradation of transcription factor IRF-1 by the ubiquitin-proteasome pathway. The C-terminal region governs the protein stability. Eur J Biochem 267: 1680-1686, 2000

32. Lecker SH, Goldberg AL and Mitch WE: Protein degradation by the ubiquitin-proteasome pathway in normal and disease states. J Am Soc Nephrol 17: 1807-1819, 2006.

33. Knecht E, Aguado C, Cárcel J, Esteban I, Esteve JM, Ghislat G, Moruno JF, Vidal JM and Sáez R: Intracellular protein degradation in mammalian cells: Recent developments. Cell Mol Life Sci 66: 2427-2443, 2009.

34. Groll M, Ditzel L, Löwe J, Stock D, Bochtler M, Bartunik HD and Huber R: Structure of 20S proteasome from yeast at $2.4 \mathrm{~A}$ resolution. Nature 386: 463-471, 1997.

35. Doherty FJ, Dawson S and Mayer RJ: The ubiquitin-proteasome pathway of intracellular proteolysis. Essays Biochem 38: 51-63, 2002.

36. Fuchs SY: The role of ubiquitin-proteasome pathway in oncogenic signaling. Cancer Biol Ther 1: 337-341, 2002.

37. Schmidt M and Finley D: Regulation of proteasome activity in health and disease. Biochim Biophys Acta 1843: 13-25, 2014

38. Liu J, Shaik S, Dai X, Wu Q, Zhou X, Wang Z and Wei W: Targeting the ubiquitin pathway for cancer treatment. Biochim Biophys Acta 1855: 50-60, 2015.

39. D'Arcy P and Linder S: Proteasome deubiquitinases as novel targets for cancer therapy. Int J Biochem Cell Biol 44: 1729-1738, 2012.

This work is licensed under a Creative Commons Attribution-NonCommercial-NoDerivatives 4.0 International (CC BY-NC-ND 4.0) License. 